\title{
A CASE
}

IN WHICH THE URACHOS REMAINED OPEN, AND A RING-SHAPED CALCULUS, FORMED UPON A HAIR IN THE BLADDER, WAS EXTRACTED THROUGH THE UMBILICUS.

BY

THOMAS PAGET, F.R.C.S. SURGEON TO THE LEICESTER INFIRMARY.

COMMUNICATED BX

WILLIAM BOWMAN, F.R.S.

Received May 20th.-Read June 11th, 1850.

The following case excited some interest at the time of its occurrence, and may be acceptable to the Royal Medical and Chirurgical Society. The specimen of calculus I send is the one referred to. It consists of a ring of lithic acid, with a fine hair in its axis; the hair being rendered visible where a projecting end has been broken off. The calculus is about as thick as a medium-sized writing quill, as shown in the subjoined figure. The history of the case is as follows :

John Conquest, ironfounder, æt. 40, has, for the last year or more, suffered from frequent and painful micturition, and on sounding I readily found a calculus. He also mentions, that upon attempting to make water, and during strong efforts at work, a portion of his urine is apt to escape at the navel which is open; and that, as far as he knows, this has been the case with him from birth.

Thus far on his admission to the Leicester Infirmary, August 15th, 1844. On subsequent examination I found 
that the nose of the catheter was easily made to appear from the bladder at the umbilical opening; and I hoped the stone, which seemed not a large one, might, without a painful operation, be extracted that way.

Towards this end the most feasible project that occurred was the distension of the bladder to its utmost with warm water, the umbilical aperture being tightly plugged, and the patient reclined upon Heurteloup's table with his head lower than the pelvis, that upon removal of the plug the calculus might flush out with the water. When, however, all was prepared, and the knife for lithotomy at hand, to be used in case of failure by other means, it occurred to me to try first a finger at the umbilicus. It readily entered, and when at full length down the unnatural passage, caught within the circle of the calculus sufficiently to enable me to drag it along the side of the bladder and extract it.

Its annular form of course led to a surmise as to what was the nucleus; and on carefully truncating it, the projecting extremity of the hair was seen. The phenomena connected with the opening at the umbilicus may be thus described.

There is a circular deficiency in the linea alba an inch in diameter, its margin being thickened and of cartilaginous hardness. Through this protrudes a hernia of the size of a goose's egg, which, in lieu of ordinary integument, is covered by mucous membrane, the surface, however, becoming dry when exposed for any length of time, as that of a vagina when inverted by complete prolapse of the uterus.

He never makes his water while the hernia is out; for when called to an effort for that purpose, the first act of the bladder is gradually to draw into the abdomen the whole of the protruded substance; its first contractions have no other effect, and it seems not to have power to force the urethra until that is accomplished. At the latter part of this act, at the instant of the disappearance of the hernia, there occurs a rather forcible jet of urine from the opening. The flow by the urethra also commences at this juncture, and the bladder is emptied in the usual way, the jet from the umbilicus ceasing, not to be renewed except by a violent 
accelerating action of the expulsor muscles. He can retain a pint of urine.

By watching the first contractions of the bladder, it becomes evident, that to the thickened margin of the umbilical aperture are attached the muscular fibres of the bladder extended along the urachus ; in fact, that the bladder and urachus are formed into a urinary receptacle, which in shape may be compared to a curved necked cupping-glass; the urethra passing out at its lower end, and its mouth being attached by muscle to the circular aperture in the linea alba. It becomes evident, also, that the pouch of the hernia is formed by evasion of the posterior part of the neck only, which is of course attached to the upper half of the aperture, and when protruded, presses upon the hard edge of the lower half sufficiently to prevent the escape of urine, except under straining efforts of the abdominal muscles.

The hernia is generally out; and he wears a girdle with a thick pad of flannel to catch the jets of water which are apt to occur while he is at work.

After the extraction of the calculus, the man was entirely relieved of his bladder symptoms; and as in consultation with my colleague and friend Mr. Nedham, it was thought unadvisable to interfere with the congenital defect, he was in a few days discharged. I saw this man at the end of April 1850. His state then was such as I have above described.

I may, in conclusion, refer to the following extract from Sir Benjamin Brodie's work on 'Diseases of the Urinary Organs,' (4th Edition, 1849, p. 263,) which notices examples of calculi formed on hairs.

"The Museum of St. George's Hospital affords two examples of calculus matter deposited on hairs. The calculi are numerous, of a peculiar oblong figure, from one third of an inch to an inch in length, with the hairs lying longitudinally in them. They are composed chiefly, if not entirely, of the phosphate of lime. One of the patients from whom these were taken was a young married lady, attended first by Mr. Wilson of Manchester, and afterwards by myself; 
and in her the origin of them seemed to be from a small congenital ovarian cyst communicating with the bladder. The other patient was an elderly lady, in whom there was no reason to believe that any ovarian disease existed. We know that hairs are occasionally found in other cysts besides those of the ovarium, and they may in this last-mentioned case have been formed in some such cyst in the kidney. I attended a gentleman who laboured under a calculus of the bladder; also under disease of the kidneys, of which last disease he died; and in whose urine I every now and then detected some very minute hairs, which I suspected to have been of renal origin. Unfortunately the body was not examined after death."

I imagine that the hair, in my own case, must have been one of the pubic hairs, which had found its way into the bladder through the umbilical opening. 